\title{
RETRACTED ARTICLE: Facilitating debugging of web applications through recording reduction
}

\section{A family of empirical studies}

\author{
Mouna Hammoudi $^{1}$. Ali Alakeel ${ }^{2}$ Brian Burg ${ }^{3}$. \\ Gigon Bae $^{4} \cdot$ Gregg Rothermel $^{1}$ (D)
}

Published online: 9 May 2017

C Springer Science+Business Media New York 2017

The Editors-in-Chief have retracted "Facilitating debugging of web applications through recording reduction" (https://doi.org/10.1007/s10664-017-9519-z) following an investigation by the University of Nebraska-Lincoln providing evidence of fabricated data used to evaluate the research results. Authors Ali Alakeel, Brian Burg, Gigon Bae, Gregg Rothermel agree to this retraction. Author Mouna Hammoudi has not responded to any correspondence from the editor or publisher about this retraction.

The online version of this article contains the full text of the retracted article as electronic supplementary material.

Electronic supplementary material The online version of this article

(https://doi.org/10.1007/s10664-017-9519-z) contains supplementary material, which is available to authorized users.

Gregg Rothermel

grother@cse.unl.edu

Mouna Hammoudi

mouna@cse.unl.edu

Ali Alakeel

alakeel@ut.edu.sa

Brian Burg

burg@cs.washington.edu

Gigon Bae

gbae@nvidia.com

1 Department of Computer Science and Engineering, University of Nebraska, Lincoln, NE 68588, USA

2 Department of Computer Science, University of Tabuk, Tabuk, Saudi Arabia

3 Department of Computer Science and Engineering, University of Washington, Seattle, WA 98105, USA

4 NVIDIA Corporation, Santa Clara, CA, USA 